\title{
Phonon-Induced Dephasing in Quantum Dots - Interpretation in Terms of Information Leakage
}

\author{
K. Roszak, P. Machnikowski and L. JaCak \\ Institute of Physics, Wrocław University of Technology \\ Wybrzeże Wyspiańskiego 27, 50-370 Wrocław, Poland
}

\begin{abstract}
We show that the phonon-induced pure dephasing of excitons in quantum dots can be interpreted in terms of information leakage from the carrier subsystem to the lattice environment. We derive a quantitative relation between the coherence of the system, as manifested by the amplitude of the coherent optical polarization, and the amount of available which path information on the system state, quantified by the distinguishability of states.
\end{abstract}

PACS numbers: 78.67.Hc, 03.65.Ta, 63.20.Kr

\section{Introduction}

Interaction between carriers in quantum dots (QDs) and phonons leads to dephasing phenomena, which manifest themselves as a partial decay of optical coherence during the first few picoseconds after an optical excitation [1]. This process is due to the spontaneous formation of a polaron-like deformation around the confined charge distribution, accompanied by emission of phonon wave packets into the bulk of the crystal $[2,3]$. These wave packets correlate (entangle) the confined carriers with the lattice of the macroscopic crystal, leading to decoherence of the quantum state of the confined carriers [4].

In general, dephasing processes are related to a trace left by a quantum system in its environment [5] which allows one, at least in principle, to infer in which of the superposed states the system really was. For instance, in the well-known two-slit experiment, the interference vanishes if the particle leaves any trace of its passage through one of the slits (due either to intentional measurement or to uncontrolled dephasing), which makes it possible to determine the path it has chosen (hence the term which path information) [6, 7]. The phonon-induced dephasing in QDs can be interpreted in the same way: since the lattice relaxation occurs only if a charge distribution is present in the QD, the presence of phonon 
packets is a trace that uniquely indicates the presence of an exciton and thus destroys the coherence between the exciton and no-exciton state in a superposition.

In this paper we show that this picture is not just a qualitative interpretation. Using the exact solution to the quantum evolution equations for the carrier-phonon system [8] we calculate the amount of information on the exciton state contained in the lattice environment (distinguishability of states [9]). We then derive a quantitative inequality which shows that the amplitude of coherent polarization in a QD is limited by the amount of information transferred to the environment.

\section{Carrier-phonon kinetics and pure dephasing}

We restrict the discussion to the ground state of an exciton confined in a QD, which is a very good approximation at low temperatures, under resonant excitation with an appropriately polarized laser beam. We will assume that the exciton wave function may be described as a product of electron and hole wave functions (which is the case for sufficiently strong confinement [3]). For simplicity, we will describe the latter as identical Gaussians with $4 \mathrm{~nm}$ width in the $x y$ plane and $1 \mathrm{~nm}$ along $z$. Under conditions assumed here, the most important dephasing effect is that of deformation potential coupling to longitudinal acoustic (LA) phonons.

The Hamiltonian of the system is therefore

$$
H=\epsilon|1\rangle\left\langle 1\left|+H_{\mathrm{ph}}+\right| 1\right\rangle\langle 1| \sum_{\boldsymbol{k}} \hbar \omega_{\boldsymbol{k}}\left(g_{\boldsymbol{k}}^{*} b_{\boldsymbol{k}}+g_{\boldsymbol{k}} b_{\boldsymbol{k}}^{\dagger}\right),
$$

where the first term describes the energy of the confined exciton ( $\epsilon$ is the energy difference between the states, including the Coulomb interaction but without phonon corrections), $H_{\mathrm{ph}}=\sum_{\boldsymbol{k}} \hbar \omega_{\boldsymbol{k}} b_{\boldsymbol{k}}^{\dagger} b_{\boldsymbol{k}}$ is the Hamiltonian of the phonon subsystem $\left(\omega_{\boldsymbol{k}}=c k\right.$ is the frequency of a phonon with wave vector $\boldsymbol{k}, c$ is the speed of sound and $b_{\boldsymbol{k}}^{\dagger}, b_{\boldsymbol{k}}$ are the corresponding creation and annihilation operators) and the third term describes the interaction, with

$$
g_{\boldsymbol{k}}=\left(\sigma_{\mathrm{e}}-\sigma_{\mathrm{h}}\right) \sqrt{\frac{1}{2 \varrho V_{\mathrm{N}} \hbar c^{3} k}} \int \mathrm{d}^{3} \boldsymbol{r} \psi^{*}(\boldsymbol{r}) \mathrm{e}^{-\mathrm{i} \boldsymbol{k} \cdot \boldsymbol{r}} \psi(\boldsymbol{r}),
$$

where $\sigma_{\mathrm{e}, \mathrm{h}}$ are deformation potential constants for electrons and holes, $V_{\mathrm{N}}$ is the normalization volume of the phonon system, $\varrho$ is the crystal density, and $\psi(\boldsymbol{r})$ are single-particle wave functions. In our calculations we use typical parameters for a self-assembled InAs/GaAs structure: $\sigma_{\mathrm{e}}-\sigma_{\mathrm{h}}=9.5 \mathrm{eV}, \varrho=5300 \mathrm{~kg} / \mathrm{m}^{3}$, $c=5150 \mathrm{~m} / \mathrm{s}$.

The Hamiltonian (1) is diagonalized by the unitary transformation $W=$ $|0\rangle\langle 0|\otimes \mathcal{I}+| 1\rangle\langle 1| \otimes w$, where $w=\exp \left[\sum_{\boldsymbol{k}}\left(g_{\boldsymbol{k}} b_{\boldsymbol{k}}^{\dagger}-g_{\boldsymbol{k}} b_{\boldsymbol{k}}\right)\right], \mathcal{I}$ is the identity operator, and the tensor product refers to the carrier subsystem (first component) and its phonon environment (second component). This allows us to find the system evolution exactly. Let us assume that an ultrashort optical pulse at $t=0$ performed a $\pi / 2$ rotation, i.e., prepared the system state $\rho_{0}=\left(\left|\psi_{0}\right\rangle\left\langle\psi_{0}\right|\right) \otimes \rho_{\mathrm{E}}$, where $\rho_{\mathrm{E}}$ is the density matrix of the phonon subsystem (environment) at thermal 
equilibrium and $\left|\psi_{0}\right\rangle=(|0\rangle+|1\rangle) / \sqrt{2}$. The system state at time $t>0$ may then be written in the form [8]:

$$
\rho(t)=\frac{1}{2}\left(\begin{array}{cc}
\rho_{\mathrm{E}} & \mathrm{e}^{\mathrm{i} E t / \hbar} \rho_{\mathrm{E}} w^{\dagger}(t) w \\
\mathrm{e}^{-\mathrm{i} E t / \hbar} w^{\dagger} w(t) \rho_{\mathrm{E}} & w^{\dagger} w(t) \rho_{\mathrm{E}} w^{\dagger}(t) w
\end{array}\right),
$$

where $E=\epsilon-\sum_{\boldsymbol{k}} \hbar \omega_{\boldsymbol{k}}\left|g_{\boldsymbol{k}}\right|^{2}, w(t)=\mathrm{e}^{-\mathrm{i} H_{\mathrm{ph}} t / \hbar} w \mathrm{e}^{\mathrm{i} H_{\mathrm{ph}} t / \hbar}$.

The density matrix for the carrier subsystem is obtained by tracing out the phonon degrees of freedom,

$$
\rho_{\mathrm{S}}(t)=\operatorname{Tr}_{\mathrm{E}} \rho(t)=\frac{1}{2}\left(\begin{array}{cc}
1 & \mathrm{e}^{\mathrm{i} E t / \hbar}\left\langle w^{\dagger}(t) w\right\rangle \\
\mathrm{e}^{-\mathrm{i} E t / \hbar}\left\langle w^{\dagger} w(t)\right\rangle & 1
\end{array}\right) .
$$

It may be shown [8] that $\left|\left\langle w^{\dagger}(t) w\right\rangle\right|=\exp \left[\sum_{\boldsymbol{k}}\left|g_{\boldsymbol{k}}\right|^{2}\left(\cos \omega_{\boldsymbol{k}} t-1\right)\left(2 n_{\boldsymbol{k}}+1\right)\right]$, where $n_{\boldsymbol{k}}$ are bosonic equilibrium occupation numbers.

Equations (3) and (4) yield an exact solution to the evolution of the whole system and of the carrier subsystem, respectively, and allow us to calculate any physical quantity of interest.

\section{Exciton dephasing and information leakage}

From Eq. (4) it is clear that phonon effects result in pure dephasing of the carrier subsystem, i.e., the occupation of the exciton state is not changed. For sufficiently regular coupling constants (as in the case of all carrier-phonon coupling mechanisms in QDs) the quantity $\left|\left\langle w^{\dagger}(t) w\right\rangle\right|$ decreases from the initial value of 1 to a certain finite value that depends on the system parameters and temperature. Since the coherent optical polarization $P(t)$ is proportional to the off-diagonal element of the reduced density matrix (4), the degree of dephasing is reflected by the optical response of the system. As a result of the joint carrierphonon kinetics, the normalized coherent polarization is partly reduced over the first few picoseconds of the system evolution according to

$$
|P(t)|^{2}=P_{0}^{2}\left|\left\langle w^{\dagger}(t) w\right\rangle\right|^{2}
$$

as shown in Fig. 1a (cf. Ref. [10]).

Using Eq. (3), we can calculate the average lattice displacement following the optical excitation of the QD, $\langle\boldsymbol{u}(\boldsymbol{r})\rangle=\operatorname{Tr}[\rho \boldsymbol{u}(\boldsymbol{r})]$, where

$$
\boldsymbol{u}(\boldsymbol{r})=\sum_{\boldsymbol{k}} \sqrt{\frac{\hbar}{2 \varrho V_{\mathrm{N}} \omega_{\boldsymbol{k}}}} \frac{\boldsymbol{k}}{k} \mathrm{e}^{\mathrm{i} \boldsymbol{k} \cdot \boldsymbol{r}}\left(b_{\boldsymbol{k}}+b_{-\boldsymbol{k}}^{\dagger}\right)
$$

is the displacement operator (for LA phonons). The result is shown in Fig. 1b. The ultrafast excitation of the carrier subsystem is followed by spontaneous lattice relaxation to a new equilibrium with a coherent polaron-like displacement field formed around the QD. At the same time, the excess energy is transmitted to the bulk of the crystal in the form of a phonon wave packet, visible in Fig. $1 \mathrm{~b}$ as a ring traveling with the speed of sound out of the QD region. By comparing Figs. 1a and $b$ one can see that the pure dephasing takes place during the time when the 

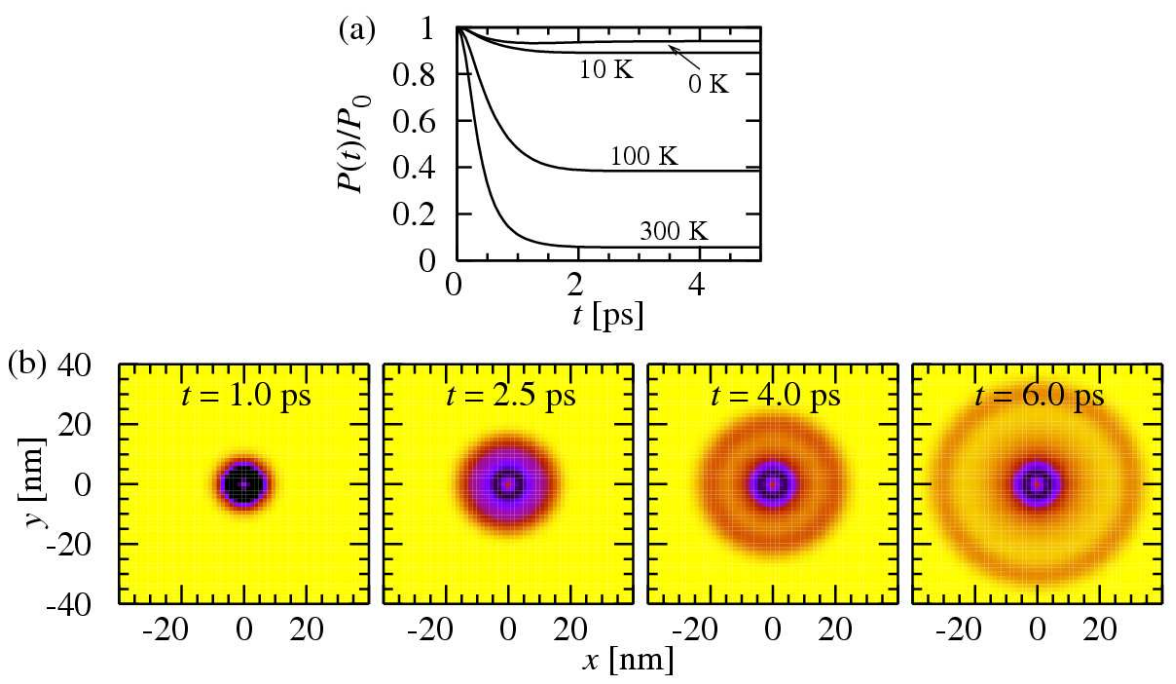

Fig. 1. (a) The decay of coherent polarization due to phonon-induced pure dephasing. (b) Lattice evolution after an ultrafast excitation. The plots show the lattice displacement in the $x y$ plane at four times after the excitation.

traveling ring is created and the plateau is reached as soon as the phonon wave packet leaves the dot.

As soon as the phonon wave packet leaves the dot the dephasing process becomes irreversible. Moreover, by radiating phonons out into the bulk of the crystal the exciton gets entangled with the macroscopic crystal lattice. This entanglement with the environment is the mechanism of the decoherence shown in Fig. 1a. On the other hand, the emitted phonon wave packet may be interpreted as a trace of the exciton presence left in its macroscopic environment. In particular, if the optical pulse excites a superposition of an empty dot and exciton states, only the latter will be accompanied by the lattice evolution. Hence, the lattice state gets correlated (entangled) with the carrier subsystem. This kind of correlation can be interpreted in terms of transfer of information (analogous to the which path information in the Young experiment) from the exciton subsystem to its environment.

This interpretation can be made quantitative with the help of a measure of information on the carrier subsystem contained in its phonon environment. The latter is defined as follows. One hypothetically performs a measurement on the environment and uses its result to predict if an exciton will be found in the QD in a subsequent measurement. The probability of a correct prediction ranges from $1 / 2$ (guessing at random in absence of any correlations) to 1 (knowing for sure, when the systems are maximally entangled). Quantitatively, an intrinsic measure is provided by the distinguishability of states $[9,11], \mathcal{D}=2(p-1 / 2)$, where $p$ is the probability for a correct prediction for the exciton state maximized over all 
possible measurements on the environment. In this way, guessing at random and knowing for sure correspond to $\mathcal{D}=0$ and $\mathcal{D}=1$, respectively.

For a general density matrix of the compound system, $\rho=$ $\sum_{i, j=0,1}|i\rangle\langle j| \otimes \rho_{i j}$, it may be shown $[8,9]$ that the distinguishability is given by $\mathcal{D}=(1 / 2) \operatorname{Tr}\left|\rho_{00}-\rho_{11}\right|$. Thus, for the carrier-phonon state of Eq. (3) one finds for the distinguishability of carrier states due to correlations with the phonon environment $\mathcal{D}(t)=(1 / 2) \operatorname{Tr}\left|\rho_{\mathrm{E}}-w^{\dagger} w(t) \rho_{\mathrm{E}} w^{\dagger}(t) w\right|$. One has, in general [12], $(1 / 4) \operatorname{Tr}^{2}\left|\rho-\rho^{\prime}\right| \leq 1-\operatorname{Tr}^{2} \sqrt{\rho^{1 / 2} \rho^{\prime} \rho^{1 / 2}}$. Hence

$$
\begin{aligned}
\mathcal{D}^{2}(t) & \leq 1-\left[\operatorname{Tr} \sqrt{\rho_{\mathrm{E}}^{1 / 2} w^{\dagger} w(t) \rho_{\mathrm{E}} w^{\dagger}(t) w \rho_{\mathrm{E}}^{1 / 2}}\right]^{2} \\
= & 1-\left[\operatorname{Tr}\left|\rho_{\mathrm{E}}^{1 / 2} w^{\dagger} w(t) \rho_{\mathrm{E}}^{1 / 2}\right|\right]^{2} .
\end{aligned}
$$

Since $\operatorname{Tr}|A| \geq|\operatorname{Tr} A|$ we may write

$$
\operatorname{Tr}\left|\rho_{\mathrm{E}}^{1 / 2} w^{\dagger} w(t) \rho_{\mathrm{E}}^{1 / 2}\right| \geq\left|\operatorname{Tr}\left(\rho_{\mathrm{E}} w^{\dagger} w(t)\right)\right|=\left|\left\langle w^{\dagger}(t) w\right\rangle\right| .
$$

Using Eq. (5) and combining Eqs. (6) and (7) we find the inequality

$$
\left[\frac{P(t)}{P_{0}}\right]^{2}+\mathcal{D}^{2}(t) \leq 1,
$$

which relates the degree of coherence to the which way knowledge of the system state.

\section{Conclusions}

We have shown that the system coherence and the amount of which path information that has been transferred to its environment are complementary quantities. The decay of coherent polarization observed in experiments may be related to the trace in the environment that allows one, at least in principle, to determine the system state. The loss of phase coherence may thus be interpreted as a result of transferring information on the system state to the lattice environment. Equation (8) reflects the fundamental complementarity that lies at the foundation of quantum theory. While phase relations pertain to wave-like properties of the quantum system, an attempt to determine the system state refers to particle-like properties of the same system, like indivisibility of the relevant entity: when measured, an exciton in a QD may be either present or absent, with no intermediate states. Here we have shown that optical experiments with semiconductor quantum dots can contribute to these fundamental issues. In this way we provide a link between the state-of-the-art experiments on semiconductor QDs and the general theory of open quantum systems.

\section{References}

[1] P. Borri, W. Langbein, S. Schneider, U. Woggon, R.L. Sellin, D. Ouyang, D. Bimberg, Phys. Rev. Lett. 87, 157401 (2001).

[2] A. Vagov, V.M. Axt, T. Kuhn, Phys. Rev. B 66, 165312 (2002). 
[3] L. Jacak, P. Machnikowski, J. Krasnyj, P. Zoller, Eur. Phys. J. D 22, 319 (2003).

[4] L. Jacak, J. Krasnyj, W. Jacak, R. Gonczarek, P. Machnikowski, Phys. Rev. B 72, 245309 (2005).

[5] W.H. Zurek, Rev. Mod. Phys. 75, 715 (2003).

[6] R.P. Feynman, R.B. Leighton, M. Sands, The Feynman Lectures on Physics, Addison-Wesley, Menlo Park, CA 1965, Vol. 3.

[7] P. Sonnentag, F. Hasselbach, Braz. J. Phys. 35, 385 (2005).

[8] K. Roszak, P. Machnikowski, Phys. Lett. A 351, 251 (2006).

[9] B.-G. Englert, Phys. Rev. Lett. 77, 2154 (1996).

[10] B. Krummheuer, V.M. Axt, T. Kuhn, Phys. Rev. B 65, 195313 (2002).

[11] G. Jaeger, A. Shimony, L. Vaidman, Phys. Rev. A 51, 54 (1995).

[12] M.A. Nielsen, I.L. Chuang, Quantum Computation and Quantum Information, Cambridge University Press, Cambridge 2000. 